\title{
Promoción de la salud emocional del pediatra de Atención Primaria
}

\author{
B. Navarro Matillas \\ Profesora colaboradora. Escuela Andaluza de Salud Pública (EASP). \\ Miembro del Grupo de Educación para la Salud, Programa de Actividades Preventivas y de Promoción \\ de la Salud de la Sociedad Española de Medicina de Familia y Comunitaria (PAPPS-SEMFYC). Granada. España.
}

\section{Resumen}

Hoy en día los profesionales de Pediatría tienen que afrontar numerosas situaciones en la consulta de Atención Primaria que les generan malestar emocional: padres que desconfían de nuestro diagnóstico; abuelos que nos recomiendan un tratamiento mejor, "como en su época se hacía"; falta de entendimiento con los directivos; presión asistencial y dificultad para organizar el trabajo; falta de reconocimiento profesional por parte de otros compañeros y de visibilidad como colectivo; etc.

Identificar y saber procesar las emociones de malestar es una habilidad personal fundamental para poder manejar estas situaciones con la mayor serenidad posible y evitar el riesgo de la constante frustración de los/as profesionales. En definitiva, todo ello va a influir de forma decisiva en el clima de la relación que se establece entre profesionales y, por lo tanto, en la capacidad del/la profesional para influir en las creencias, emociones y comportamientos de las personas con las que se relaciona o que están bajo su dirección.

En este taller se van a abordar, en primer lugar, algunas ideas básicas acerca de cómo funcionan las emociones humanas, para, en segundo lugar, ofrecer algunas estrategias dirigidas a procesarlas y controlarlas. Vamos a centrarnos exclusivamente en el funcionamiento y manejo de las emociones desagradables, ya que suelen tener una influencia decisiva en la capacidad de las/os profesionales para establecer una relación positiva y eficaz que pueda estimular la motivación de los/as otros/as a seguir pautas de comportamiento.

Además, se aportarán ciertos verbos que en la comunicación con los padres, familiares, jefes y compañeros facilitan la comprensión, el entendimiento, el respeto y la confianza mutua.

Palabras clave: Habilidades. Emociones. Distorsiones cognitivas. Reestructuración cognitiva. Comunicación. Verbos clave.

Bibiana Navarro Matillas: bibiana.navarro.matillas@gmail.com

La autora declara no presentar conflictos de intereses en relación con la preparación y publicación de este artículo.

Texto basado en: Bimbela Pedrola JL, Navarro Matillas B. Cuidando al formador. Habilidades emocionales y de comunicación. 2. ${ }^{a}$ edi-

ción. Granada: EASP; 2007. 


\section{Promotion of the Primary Care pediatrician's emotional health}

\section{Abstract}

Pediatric professionals are faced today with numerous situations in Primary Care, which generate emotional distress: parents who are suspicious of our diagnosis, grandparents who tell us a better treatment, "as in their days was done", lack of understanding with management, workload and difficulty in organizing work, lack of professional recognition by peers and visibility as a group, etc.

Identifying and learning processing the emotional discomfort is a fundamental personal ability to handle these situations with the greatest serenity possible and avoid the risk of a continued frustration of the professionals. In short, all of it is going to make a decisive impact on the climate of the relationship established among professionals, and therefore the ability of the professional to influence beliefs, emotions and behavior of the people with whom he/she relates or are under their direction.

In this workshop, some basic ideas about how human emotions work will be addressed in the first place, and secondly some strategies to processes and control them will be offered. We will focus exclusively on the operation and handling of unpleasant emotions, as they often have a decisive influence on the ability of the professionals to establish a positive and effective relationship that can stimulate the motivation of the others to follow behavior patterns.

Moreover, some verbs that make easier the comprehension, understanding, respect and mutual confidence will be provided.

Key words: Skills. Emotions. Cognitive distortions. Cognitive restructuration. Communication. Key verbs.

\section{Habilidades emocionales}

\section{Cómo funcionan las emociones humanas}

La capacidad humana para sentir determinadas emociones forma parte del equipaje biológico necesario para la supervivencia de la especie. La sensibilidad humana a las emociones tiene como principal objetivo señalar qué hechos son verdaderamente importantes para el bienestar y la vida, ya sea por su peligrosidad o por su potencial placentero.

Además, las emociones tienen otra relevante función: empujar hacia la ac- ción, ya sea para terminar con el peligro o el malestar, ya sea para acercarse y mantener la fuente de bienestar. De hecho, son seguramente la fuente de información que más peso tiene a la hora de tomar de decisiones.

Sin embargo, no siempre las emociones cumplen esta funcionalidad, y cuando esto ocurre pasan de ser un recurso inestimable a ser una fuente de intenso malestar, desorganizando a su vez la capacidad para la acción

Las habilidades de autocontrol emocional tienen como principal objetivo evitar que el malestar sea demasiado in- 
tenso $y$, sobre todo, que se alargue en el tiempo, que se vuelva crónico. Evitando estas dos circunstancias, el malestar puede funcionar como una señal de aviso para poner en marcha estrategias de afrontamiento dirigidas bien a actuar para cambiar o terminar con la situación que genera malestar, bien para tratar de adaptarse a ella.

\section{Cómo se pueden manejar las emociones}

Una de las vías para tratar de disminuir el malestar que una persona siente ante determinadas situaciones es identificar cómo está valorando e interpretando esa situación (qué piensa sobre ella) $y$, si existen distorsiones, reestructurarlas para que sean más ajustadas a la situación y generen menos malestar. En este apartado se describirán, en primer lugar, algunas de las distorsiones cognitivas más frecuentes, para a continuación, proponer un ejercicio que permite identificarlas y modificarlas (reestructuración cognitiva).

\section{Distorsiones cognitivas}

Dentro de la Psicología, las corrientes cognitivistas afirman que existe una serie de distorsiones cognitivas que serían las responsables de que ante determinadas situaciones las personas se vieran afectadas por alteraciones emocionales intensas o crónicas, influyendo de manera poderosa en el bienestar de las personas y en su capacidad para tomar decisiones en consonancia con sus necesidades y objetivos ante las situaciones a las que tienen que enfrentarse.

Desde las teorías psicosociales del estrés, también se afirma que este fenómeno se produce cuando las personas tienen que enfrentarse a situaciones que valoran como peligrosas o dañinas, $\mathrm{y}$ ante las cuales perciben que no tienen recursos para poder afrontarlas.

Siguiendo estas dos aportaciones teóricas, a continuación se describen algunas distorsiones cognitivas cuyos efectos principales serían el de aumentar la percepción de que la situación que se está viviendo es amenazante, desagradable o dañina, y el de disminuir la percepción de que se tienen recursos para poder enfrentarla.

a) Filtro mental: percibir solo lo negativo (los problemas, las dificultades, las limitaciones) y no percibir y valorar lo positivo (aquellas áreas en las que no se tienen problemas o limitaciones y disfrutar de ellas). Percibir solo lo positivo también sería una distorsión, pero en nuestra cultura es más frecuente que el filtro mental no permita valorar lo posi- 
tivo. A esta distorsión también se la conoce como "abstracción selectiva".

b) Las etiquetas: convertir un comportamiento propio o ajeno en un rasgo de personalidad y juzgarlo negativamente. Las etiquetas son distorsiones porque son una sobregeneralización y convierten la parte en el todo. En nuestra cultura es relativamente frecuente atribuir los comportamientos de las personas con las que nos relacionamos, especialmente si se juzgan como negativas, a rasgos de personalidad ("es así"), en vez de atribuirlos a una constelación de factores complejos como las creencias, los deseos, elementos del contexto y de la situación, las emociones, o el propio comportamiento de la persona a la que se está etiquetando.

c) Los "siempre-todo-nunca-nada": junto con las etiquetas, representan también pensamientos sobregeneralizados. Los pensamientos de este tipo de pensamiento "casi nunca" se ajustan a la realidad; sin embargo, hacen sentir a la persona como si la realidad fuera así.

d) Adjetivos desmedidos: dramatizar las situaciones utilizando adjetivos desmesurados como "horroroso" o "insoportable".

e) Pensamientos anticipatorios improductivos: prever de antemano que van a ocurrir eventos o situaciones negativas, sin pensar en cómo podrían prevenirse o cómo podrían paliarse sus consecuencias, si llegarán a ocurrir.

f) Lectura mental: tener la certeza de que se sabe lo que otras personas están pensando, sintiendo o deseando, sin tener pruebas objetivas para ello.

g) Falacia de control por exceso: pensar que se tiene todo el control y, por lo tanto, toda la responsabilidad sobre determinados sucesos, cuando no es así.

h) Falacia de control por defecto: pensar que no se tiene ninguna responsabilidad y, por lo tanto, ningún control sobre determinados sucesos, cuando no es así.

\section{Reestructuración cognitiva}

Este ejercicio se realiza de forma escrita ya que, en caso contrario, es difícil analizar los pensamientos. Resulta especialmente útil cuando una persona (ya sea un/a profesional o un/a jefe/a) ha de enfrentarse a situaciones que se repiten con frecuencia y que le generan malestar, pero que, a pesar de ello, no ha conseguido aprender a enfrentarse a ellas de una forma más calmada.

A continuación se van a describir los pasos necesarios para reestructurar o modificar cogniciones distorsionadas.

1. Describir la situación que genera malestar. Es necesario tratar de relatar, 
lo más objetivamente posible, los hechos que generan malestar, y diferenciarlos de la interpretación o valoración subjetiva (nivel cognitivo) que se hace de esos hechos.

Por ejemplo, en el caso de un profesional, escribir que "el responsable del establecimiento no me hace caso", es una interpretación subjetiva de los siguientes hechos: "El responsable del establecimiento no sigue las indicaciones".

Esta confusión entre la situación (los hechos) y el nivel cognitivo (la valoración subjetiva que se hace de esos hechos) es una de las principales dificultades que se presentan a la hora de tratar de modificar o reestructurar el nivel cognitivo, ya que se está presuponiendo que no existe valoración subjetiva. También es muy importante centrarse en una sola situación, y no en un conjunto de ellas.

Situación: el responsable del niño dice que ha seguido todas las indicaciones pero los análisis indican que los niveles de $\mathrm{X}$ han sido muy elevados durante los últimos meses.

\section{2. ¿Cómo me siento ante esa situa-} ción? Identificar y escribir el tipo de emociones que se presentan cuando se da la situación descrita. En nuestra cultura, estamos poco acostumbrados a estar en contacto con nuestras emociones, por lo que es frecuente que las personas digan cosas como "me siento fatal", en vez de emociones concretas como "triste", "enfadada" o "frustrada". Sin embargo, es importante identificarlas, darles nombre, para entender primero cómo se está viviendo una situación y poder luego controlar esas vivencias.

Lo más frecuente es que la misma situación genere emociones distintas en cada persona, ya que no están producidas solo por la situación, sino también por la interpretación subjetiva de esa situación.

¿Qué sienten algunas/os profesionales cuando un padre/madre/abuela /abuelo dice que ha seguido todas las indicaciones pero los análisis indican que los niveles de $X$ han sido muy elevados durante los últimos meses?

Emociones: indignación, impotencia y frustración

3. ¿Qué pienso? Identificar los pensamientos que aparecen ante la situación. Es frecuente también encontrarse con que las personas tienen dificultades para identificar cómo están valorando determinada situación. Una forma de facilitar este paso es tratar de responder a la siguiente pregunta ¿Por qué me siento indignada? 
Pensamientos (tabla 1):

"Me está mintiendo, me quiere engañar; ¿se cree que soy imbécil?" (indignación).

"Si no cambia de actitud, yo no puedo hacer nada" (impotencia)

"Estoy harta de esto. Siempre lo mismo; mis esfuerzos no sirven para nada" (frustración).

\section{Identificar las distorsiones cogniti-} vas que puede haber en los pensamientos: "Me está mintiendo, me quiere engañar; ¿se cree que soy imbécil?" (lectura mental); "Si no cambia de actitud, yo no puedo hacer nada" (falacia de control por defecto); "Estoy harta de esto. Siempre lo mismo; mis esfuerzos no sirven para nada" (sobregeneralización y falacia de control por exceso).

\section{5. ¿Cómo puedo valorar esta situación} de una forma menos distorsionada? Reestructurar los pensamientos distorsionados, cuestionando su validez y tratando de cambiarlos por otros más adaptados a la situación. Es crucial que esta nueva valoración de la situación, los nuevos pensamientos, resulten creíbles para la persona que realiza el ejercicio.

6. ¿Cómo me sentiría ante esta misma situación si mis pensamientos fueran los segundos en vez de los primeros?

Emociones: cierta incomodidad, duda, sensación de control, tranquilidad. Como es evidente, la actuación va a ser más ajustada a la situación si se basa en cogniciones que generan emociones como la incomodidad, la duda, la sensación de control y la tranquilidad, que si

Tabla 1. Pensamientos distorsionados y no distorsionados

\begin{tabular}{l|l}
\hline Pensamientos distorsionados & Pensamientos no distorsionados \\
\hline $\begin{array}{l}\text { "Me está mintiendo, me quiere engañar; } \\
\text { ¿se cree que soy imbécil?" }\end{array}$ & $\begin{array}{l}\text { "Creo que lo que está diciendo no es cierto, o por } \\
\text { lo menos no encaja con lo que dicen las pruebas" }\end{array}$ \\
nada" & $\begin{array}{l}\text { "Puede ser que no se atreva a decírmelo. } \\
\text { La verdad es que no sé lo que está pasando" } \\
\text { "Yo sí puedo hacer algo para tratar de aclarar esta } \\
\text { situación; por ejemplo, preguntarle a qué atribuye } \\
\text { él los resultados o plantearle la necesidad } \\
\text { de apuntar los pasos que realiza" }\end{array}$ \\
\hline "Estoy harta de esto. Siempre lo mismo; & "Claro que sirven mis esfuerzos; lo veo en muchas \\
mis esfuerzos no sirven para nada" & $\begin{array}{l}\text { de mis intervenciones. De todas formas es } \\
\text { importante recordarme de vez en cuando que el } \\
\text { cumplimiento de las pautas no solo depende de } \\
\text { mis esfuerzos; también se tienen que esforzar los } \\
\text { responsables de los establecimientos, y es normal } \\
\text { que les cueste seguir todas las indicaciones" }\end{array}$ \\
\hline
\end{tabular}


se basa en pensamientos distorsionados que producen emociones como la indignación, impotencia y frustración.

\section{Verbos y conceptos claves}

\section{en comunicación}

\section{Preguntar}

Este es un verbo fundamental para que la/el profesional evite los errores derivados de dar algo por supuesto, y obtenga la información necesaria para diseñar una intervención más eficaz. Además, si se realiza con la "música" adecuada, resulta muy motivador y estimulante para la persona.

Trasmite a la persona interés (por su salud, sus opiniones, sus temores, sus sentimientos...) y ello, como bien se sabe, aumenta enormemente la confianza entre la/el profesional y la persona con la que se relaciona o está bajo su dirección.

Facilita la participación y el protagonismo de la persona con la que se relaciona o está bajo su dirección, lo cual ayuda a mejorar considerablemente su motivación y su compromiso en el proceso.

\section{Escuchar... activamente}

Tanto el lenguaje verbal como el no verbal ayudan a realizar una buena es- cucha activa. El primero mediante "letras" como: pedir alguna aclaración a lo dicho por la persona, solicitar la repetición de algún comentario, parafrasear, etc. El segundo mediante "músicas" como: mirar (no agresivamente) a los ojos de la persona, asentir con la cabeza, incorporarse en la silla acercándose a la persona, etc.

\section{Empatizar... activamente}

Las "traducciones" más frecuentes y aceptadas de este verbo son: "ponerse en el lugar de la otra persona", "entender sus sentimientos, sus razones, sus miedos, sus temores". Aun en esos casos más difíciles vale la pena que la/el profesional haga el esfuerzo de lograr una buena empatía, pues ello va a resultar muy importante para establecer una comunicación con la otra persona que se base en la confianza y el respeto mutuos y que consiga el acercamiento emocional y el subsiguiente compromiso por parte de la otra persona y un aumento de la cantidad y calidad de la información aportada por ella/él.

\section{Resumir/sintetizar}

A lo largo de la comunicación interpersonal, es importante ir dando "puntos de anclaje" que permitan, por una parte, seguir el hilo conductor y asimilar 
los mensajes en él contenidos $y$, por que sea la propia persona con la que otra, identificar los distintos puntos cla- nos comunicamos (el interlocutor) ve, lo más relevante. Si eso es importan- quien resuma/sintetice las ideas clave, te hacerlo durante todo el periodo en el pues si lo hace ella/él misma/o, la/el que se produce la comunicación, resulta profesional podrá comprobar el grado fundamental al final de la conversación, de comprensión, y por tanto tendrá inen el momento del "cierre". En la medi- formación "de primera mano" para da de lo posible va a ser más interesante evaluar ese proceso de comunicación. 